\title{
A Rare Case of Sublingual Gland Hydatid Cyst
}

Shima Hajibegloo ${ }^{1}$ (D) Farrokh Heidari $^{1}$ (D), Amirhossein Yadegar $^{1}$, Firouzeh Heidari ${ }^{1}$ (D) Ebrahim Karimi $^{1}$, Keyvan Aghazadeh $^{1 *}$ (D)

1. Otorhinolaryngology Research Center, Tehran University of Medical Sciences, Tehran, Iran

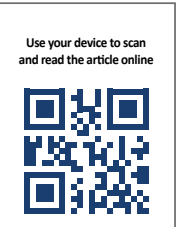

Citation Hajibegloo Sh, Heidari F, Yadegar A, Heidari F, Karimi E, Aghazadeh K. A Rare Case of Sublingual Gland Hydatid Cyst. Case Reports in Clinical Practice. 2019; 4(4):115-118.

Running Tite A Rare Case of Sublingual Gland Hydatid Cyst

\section{(c) (1) (3)}

Article info:

Received: 02 November 2019

Revised: 20 November 2019

Accepted: 05 December 2019

Keywords:

Hydatid cyst; Sublingual gland; Case report

\section{ABSTRACT}

Background: Hydatid cyst is a zoonotic disease due to the infection with the larvae of Echinococcus granulosus. The liver and lungs (80\%) are the main organs involved and rarely head and neck.

Case Presentation: A 47-year-old Iranian man presented to our center complaining of sublingual inflammation for 8 months. Sonography revealed a cystic lesion of $4 \times 5 \times 6 \mathrm{~cm}$ within the tongue. CT-scan showed a well-defined and hypodense mass in the floor of the mouth. Cyst resection was performed with the probability of ranula. Pathology was suggestive of hydatid cyst. Further workups revealed liver involvement. Therefore, he was treated with albendazole for 2 months.

Conclusion: The hydatid cyst in the head and neck is rare and the involvement of sublingual gland is extremely rare. However, in endemic areas, hydatid cyst should be considered in the differential diagnosis of head and neck masses.

\section{Introduction}

chinococcosis is a zoonotic disease caused by the larval stage (hydatid cyst) of tapeworms. It is endemic in the Mediterranean, Middle East, Asia, South America, and Southeast Af- rican countries [1]. The canines (dog, fox, and wolf) are its definite hosts, whereas human is an accidental host. Humans are affected by eating food and drinking water contaminated with the eggs or definite host stool [2, $3]$. The most common sites of the involvement are liver and lungs (80\%) [2]. In endemic regions, $5-15 \%$ of all

* Corresponding Author:

Keyvan Aghazadeh, MD.

Address: Otorhinolaryngology Research Center, Tehran University of Medical Sciences, Tehran, Iran.

E-mail: aghazadeh@sina.tums.ac.ir 
Echinococcosis infections are associated with musculoskeletal and soft tissue involvement [4]. Early head and neck involvement even in endemic areas is very rare (1-2\%) [2-4]. In addition, the sublingual involvement is extremely rare. We reported this form of hydatid cyst in the present case study.

\section{Case Presentation}

A 47-year-old man presented to our center complaining of the inflammation in the mouth floor and sublingual swelling lasted for about 8 months. Swelling has increased over the past 3 months. He had a mild pain in the sublingual area. He did not report any history of fever, chills, anorexia, and weight loss and also had no significant past medical history and taking drugs. On physical examination, he had a clear swollen mouth without tenderness. The mass was soft with no evidence of inflammation and erythema. We obtained an aspiration, which revealed a colorless liquid. The cytology examination reported no clear diagnosis. Then, we performed sonography that showed a cystic lesion of $4 \times 5 \times 6 \mathrm{~cm}$ within the tongue with the probability of an epidermoid cyst. CT scan revealed a well-defined hypodense mass in the floor of the mouth (Figure 1).

Our patient underwent excision with a probable diagnosis of ranula. The macroscopic appearance of the lesion was a cyst with a thick and white oyster wall (Figure 2). The pathology test confirmed the hydatid cyst.

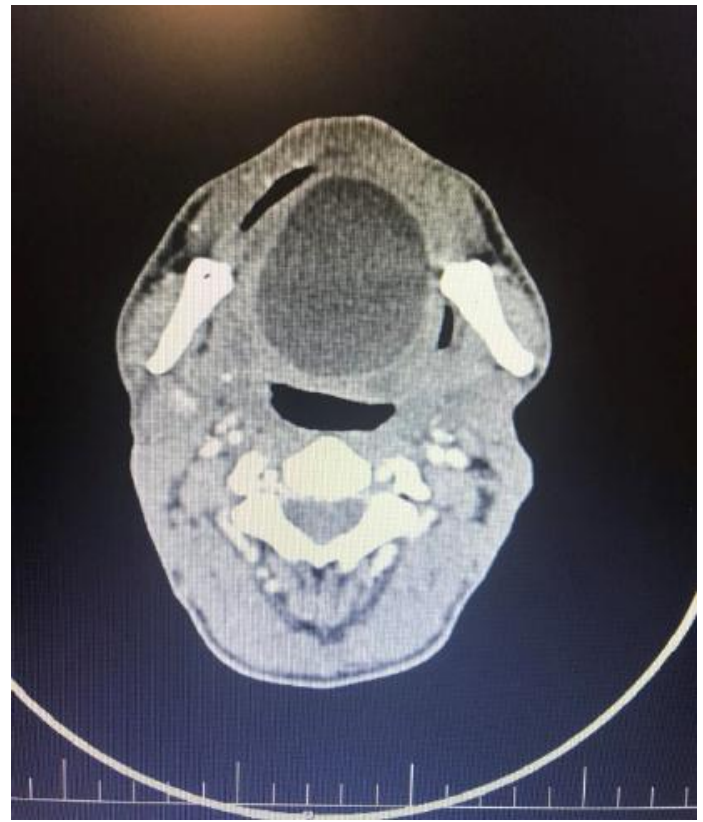

CRCP

Figure 1. CT scan with IV contrast showing a sublingual well-defined hypodense mass
We then performed a thoracoabdominopelvic CT scan with IV contrast, which showed a similar lesion in the right lobe of liver (Figure 3). No remarkable lesions were found in the lungs and chest. Laboratory studies found a positive hydatid IgG antibody as well as the negative IgM antibody. Eventually, he was treated with albendazole (400 mg) for two months and followed-up regularly.

\section{Discussion}

E. granulosus is the causative agent of the hydatid cyst. The life cycle of this parasite involves two hosts: the primary and the intermediary hosts. In the primary host (dog and other canines), the puberty or reproduction of parasite occurs, whereas in the intermediary host, the larval stage or reproduction of parasite is occurred [5]. An individual, who is infected by eating food or drinking water contaminated with matured eggs, is considered an accidental host [5]. After ingestion, the parasite perforates the wall of small intestine and moves to the liver and other organs through the bloodstream. The larvae may be trapped in capillaries of various organs in their pathways and cause inflammatory responses. Few of them can survive to form cysts with thick, slow-growing walls [5].

Other mechanisms for spreading are the systemic release through the lymph nodes and direct spread toadjacent sites [6]. Diagnosis of hydatid cyst before the operation is important, because the content of thee

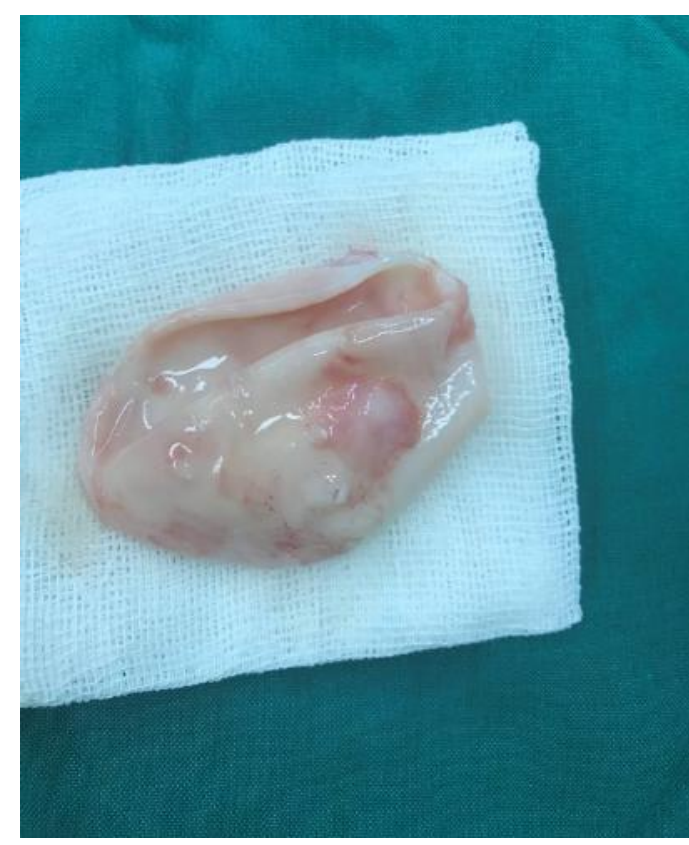

CRCP

Figure 2. Macroscopic appearance of the cyst after surgery 


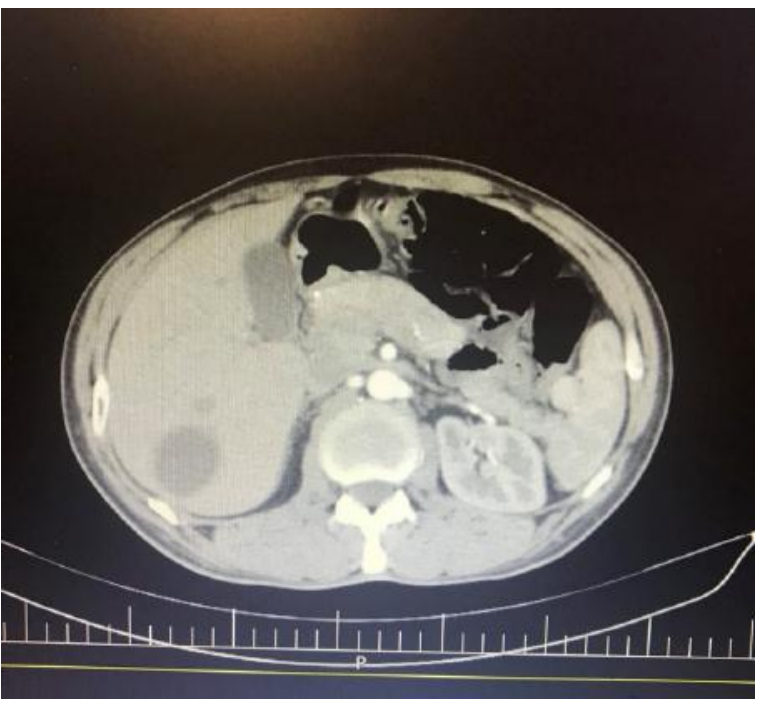

CRCP

Figure 3. Hepatic multiple well-defined hypodense cysts with different sizes in the coronal section on abdominal CT scan with IV contrast

ruptured cyst may be released during the resection, and the risk of anaphylactic shock will increase [6]. The best diagnostic modalities are sonography, CT scan, Magnetic Resonance Imaging (MRI), serologic tests (Enzyme-Linked Immunosorbent Assay (ELISA), Casoni test, latex agglutination and direct hemagglutination, Fine Needle Aspiration (FNA), and histopathology [1]. Sonography is the primary and selective diagnostic modality, especially in the diagnosis of female cysts and hydatid cyst suspended parts [5-7].

CT scan and MRI clearly show the cyst wall defects and also complications, such as rupture and cyst infection. Asparagus and FNA report a transparent liquid without malignant cells suggesting a benign cyst [6]. The hydatid cyst is usually asymptomatic. The presence of symptoms depends on the location, size and pressure to the adjacent tissues [1]. The most common sites of the hydatid cyst are liver and lungs. The involvement of head and neck is rare and involvement of the sublingual glands is extremely rare $[8,9]$.

In 2008, another case was reported in India who was a 23-year-old man with swelling in the midline of the sublingual region. The appearance of hydatid cyst was similar to our patient, i.e. a soft mass without tenderness and inflammatory symptoms. The clinicians performed a surgical procedure with a possible diagnosis of ranula and dermoid cyst. Pathology examination showed hydatid cyst [5]. In addition, rare cases have been reported in the parotid gland, submandibular gland, buccal mucosa and other areas in the oral cavity (cheek) [2, 5-7].

For treatment, the dashing parasitic quantum group, such as albendazole and mebendazole are beneficial. Complete cyst resection alone is an effective treatment for hydatid cyst and it appears to be a standard treatment in cases who do not respond to medication [6]. For several large cysts, surgery is not suitable and chemotherapy can be used. When the contents of the cyst during the operation came out, they should be removed and the cyst should be washed with an antiseptic solution [8].

However, the pathological examination is preferred after resection of the cyst. In all patients, the regular follow-up is necessary to make sure of definitive treatment and no recurrence [1].

The prevalence of hydatid cyst in head and neck areas is extremely rare, especially in sublingual glands. However, it should be considered in the differential diagnosis of the head and neck masses in endemic areas.

\section{Ethical Considerations}

\section{Compliance with ethical guidelines}

The research was carried out according to the principles of the Declaration of Helsinki. Informed consent was taken from the patient.

\section{Funding}

This research did not receive any specific grant from funding agencies in the public, commercial, or not-forprofit sectors.

\section{Conflict of interest}

The authors declared no conflict of interest.

\section{References}

[1] Karaman E, Yilmaz M, Ada M, Yilmaz RS, Isildak H. Unusual location of primary hydatid cyst: Soft tissue mass in the parapharyngea region. Dysphagia. 2011; 26(1):75-7. [DOI:10.1007/s00455-0109278-0] [PMID]

[2] Arora VK, Chopra N, Singh P, Venugopal VK, Narang S. Hydatid cyst of parotid: Report of unusual cytological findings extending the cytomorphological spectrum. Diagnostic Cytopathology. 2016; 44(9):770-3. [DOI:10.1002/dc.23515] [PMID] 
[3] Alam M, Hasan SA, Hashmi SF. Unusual presentation of hydatidosis - neck lump causing costo-vertebral erosion. Iranian Journal of Otorhinolaryngology. 2016; 28(88):363-7. [PMCID] [PMID]

[4] Mohindra S, Naidu SK, Bal A, Kumar M. Primary hydatid cyst presenting as a mass in the supraclavicular region: An unusual case report and literature review. Journal of the Pakistan Medical Association. 2013; 63(10):1309-11. [PMID]

[5] Jumani KT, Ananthamurthy A, Joy B, Nayar R. Sublingual hydatid cyst: Case report and literature review. The Journal of Laryngology \& Otology. 2009; 123(1):e6. [DOI:10.1017/S0022215108003757] [PMID]

[6] Hossain MF, Rahman M, Amin R, Ahmed T, Karmaker A. Hydatid cyst of the submandibular region. Official Journal of Khwaja Yunus Ali Medical College. 2017; 5(1):480-3. [DOI:10.3329/kyamcj. v5i1.32321]

[7] Alaparthi RK, Yelamanchili S, Nunsavathu PN, Sode U. Intraoral hydatid cyst: A rare case report. Journal of Indian Academy of Oral Medicine and Radiology. 2015; 27(3):457-60. [DOI:10.4103/09721363.170486]

[8] Lavanya RM, Kamath VV, Komali Y, Krishnamurthy Sh. Hydatid cyst of the buccal mucosa: An unusual presentation. Indian Journal of Dentistry. 2015; 6(3):157-60. [DOI:10.4103/0975-962X.160350] [PMID] [PMCID]

[9] Diwan R, Mogra N, Purohit M. Primary hydatid cyst of the parotid gland. BMJ Case Reports. 2015; pii:bcr2014209217. [DOI:10.1136/ bcr-2014-209217] [PMID] [PMCID] 